\title{
Ballistic and quasiballistic tunnel transit time oscillators for the terahertz range: Linear admittance
}

\author{
Z. S. Gribnikov \\ Department of ECE, Wayne State University, Detroit, Michigan 48202 and Department of EECS, \\ University of Michigan, Ann Arbor, Michigan 48109
}

N. Z. Vagidova) and V. V. Mitin

Department of ECE, Wayne State University, Detroit, Michigan 48202

G. I. Haddad

Department of EECS, University of Michigan, Ann Arbor, Michigan 48109

(Received 21 November 2002; accepted 13 February 2003)

\begin{abstract}
We have considered interactions between ballistic (or quasiballistic) electrons accelerated by a dc electric field in an undoped transit space (T space) and a small ultrahigh frequency ac electric field and have calculated the linear admittance of the $\mathrm{T}$ space. Electrons in the $\mathrm{T}$ space have a conventional, nonparabolic dispersion relation. After consideration of the simplest specific case when the current is limited by the space charge of the emitted electrons, we turned to an actual case when the current is limited by a heterostructural tunnel barrier (B barrier) separating the heavily doped cathode contact and the $\mathrm{T}$ space. We assumed that the $\mathrm{B}$ barrier is much thinner than the $\mathrm{T}$ space and both dc and ac voltages drop mainly across the $T$ space. The emission tunnel current through the $\mathrm{B}$ barrier is determined by the electric field $E(0)$ in the $\mathrm{T}$ space at the boundary $\mathrm{B}$ barrier/T space. The more substantial is, the tunnel current limitation the higher the electric field $E(0)$ becomes. We have shown that for a space-charge limited current the change from parabolic dispersion to the nonparabolic branch induces narrowing and closing of the frequency windows of transit-time negative conductance starting with the lowest-frequency windows. These narrowing and closing frequency windows become effective only for very high voltages $U$ across the $\mathrm{T}$ space: $U \gg m V_{S}^{2} / 2 e$, where $m$ is the effective mass for the parabolic branch and $V_{S}$ is the saturated velocity for the nonparabolic branch. For moderate voltages $U$, the effects of nonparabolicity are not very substantial. The tunnel current limitation decreases the space-charge effects in the $\mathrm{T}$ space and diminishes the role of the detailed electron dispersion relation. As a result, restoration of the frequency windows of transit-time negative conductance and an increase in the value of this negative conductance occur. The implementation of the considered tunnel injection transit time oscillator diode promises to lead to efficient and powerful sources of terahertz range radiation.

(c) 2003 American Institute of Physics. [DOI: 10.1063/1.1565496]
\end{abstract}

\section{INTRODUCTION}

Tunnel injection transit-time oscillator diodes have been suggested $^{1-3}$ as the highest-speed transit-time devices intended to dominate at the highest frequencies that are unreachable for ordinary IMPATT diodes. However, the passing of time has shown ${ }^{4,5}$ that up to now solid-state tunnel injection oscillators have not justified these expectations and they yield to IMPATT diodes in all the measurable frequency ranges.

Here, we consider and substantiate theoretically a version of tunnel injection transit-time diodes (TUNNETTs) that differ noticeably from the traditional ones. As the traditional version, which we use for comparison, we select one of the best GaAs TUNNETTs, which has been described in detail in Refs. 6-8. Below, we call this version the traditional diode (TD). The main peculiarities of the present diode (PD) in comparison with the TD are as follows.

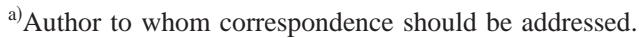

(1) The PD is an $n$-type unipolar heterostructural diode. It does not contain any acceptor-doped regions nor any $p$-type contacts. The TD is a typical $p^{+} n$-type diode. ${ }^{6-8}$

(2) Electron injection into a transit space of the PD is realized by electron tunneling through a moderately high square heterobarrier. It takes place in moderately strong electric fields $\left(E \leqslant 10^{5} \mathrm{~V} / \mathrm{cm}\right)$. In the TD, the analogous injection is realized by interband (Zener) tunneling. In the GaAs case it requires electric fields that noticeably exceed $10^{6} \mathrm{~V} / \mathrm{cm}$ and corresponding voltages across the diode of up to $9 \mathrm{~V}$.

(3) The PD has a very short transit space with a length $l \leqslant 50-100 \mathrm{~nm}$. The material for this transit space is selected to provide ballisticity or quasiballisticity for an electron transported through this space at a velocity $\leqslant 10^{8} \mathrm{~cm} / \mathrm{s}$. In the TD, all the regions including the transit space are GaAs grown with low-lying electron $L$ and $X$ valleys. Therefore, in a relatively short transit space of the TD with $l \approx 300 \mathrm{~nm},{ }^{6-8}$ there is diffusive electron transport with a drift velocity $\sim(1-2) \times 10^{7} \mathrm{~cm} / \mathrm{s}$. 


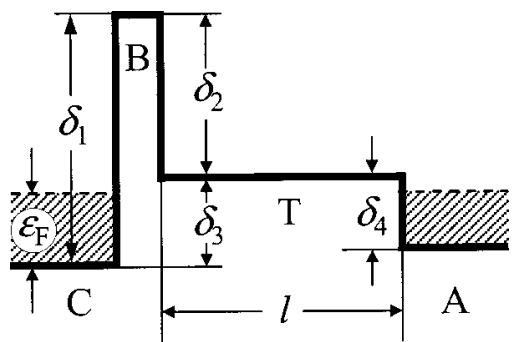

FIG. 1. Structure of the present tunnel injection transit-time diode (PD). $\mathrm{C}-\mathrm{a}$ heavily doped cathode contact; $\mathrm{B}-\mathrm{a}$ tunnel emitter heterostructural barrier; $\mathrm{T} — \mathrm{a}$ transit space; $\mathrm{A}-\mathrm{a}$ heavily doped anode contact.

Some elements of the proposed PD (such as unipolarity and a heterobarrier electron injector) were realized in the so-called HEBITT diode ${ }^{9}$ (heterobarrier-injection transittime diode), but the selected parameters of the heterobarrier, as well as a transit space that was too long, allowed the writers of Ref. 9 to reach only a $60 \mathrm{GHz}$ oscillation limit.

Below, we describe a schematic sketch of the PD design including possible semiconductor materials for its implementation (Sec. II). Then, we describe the theoretical model and the dc regime (Sec. III). The central part of our article is contained in Sec. IV, in which a linear theory of the interaction of an ultrahigh-frequency electric field with the electron current is stated and a negative conductance for terahertz and superterahertz frequencies is predicted. Section V is devoted to a discussion followed by concluding remarks.

\section{DESIGN SKETCH}

We present a sketch of the PD design in Fig. 1. It should be implemented on the basis of four material regions: $\mathrm{C}$ (cathode contact, $\mathrm{C}$ contact), A (anode contact, A contact), B (tunnel heterobarrier, $\mathrm{B}$ barrier), and $\mathrm{T}$ (transit space, $\mathrm{T}$ space). The last is the most critical part of the device: here electrons gain all their kinetic energy and transform a certain part of this gained energy into energy of ultrahigh-frequency electric oscillations. We need to realize ballistic or quasiballistic high-energy electron transport just in the T space. Although most of the dc voltage drops across the $\mathrm{T}$ space, the electric current is controlled by the electric field in the thin B barrier, across which only a small part of the voltage drops, and which should be an effective current injector (together with the $\mathrm{C}$ contact). The A contact also plays a very important role. It must accept the high-energy ballistic electrons coming from the $\mathrm{T}$ space both without quantum-mechanical reflection and without backscattering. This is necessary because backscattered electrons can substantially change the transport characteristics of the ballistic device, transforming the ballistic transport into a quasidiffusive one. Therefore, the anode contact A should not be a typical material system. Neither the $\mathrm{C}$ contact nor the A contact should absorb the ultrahigh-frequency radiation emitted by the traveling electrons.

Restricting the list of materials for the $\mathrm{T}$ space to only the zinc blende $A_{\mathrm{III}} B_{\mathrm{V}}$ structures, only the ones with In as the anion (that is, $A_{\mathrm{III}}=\mathrm{In}$ ) are suitable since all the others have low-energy positioned $L$ and (or) $X$ valleys with a high rate of $\Gamma$-electron scattering into them. Let us list several such materials: InP, $\operatorname{In}_{0.53} \mathrm{Ga}_{0.47} \mathrm{As}$ (on an InP substrate), $\mathrm{InP}_{0.69} \mathrm{Sb}_{0.31}$ (on an InAs substrate), InAs, and InSb. The first is the most attractive. In this case, $L$ valleys have the most reliable separation ${ }^{10}\left(\varepsilon_{L} \cong 0.6 \mathrm{eV}\right.$ above the $\Gamma$-valley bottom). There are several other more optimistic estimates for $\varepsilon_{L}: 0.7,{ }^{11} 0.832$, and $0.86 \mathrm{eV}^{12} X$ valleys always lie higher in accordance with all the estimates (for example, ${ }^{10} \varepsilon_{X}$ $\cong 0.96 \mathrm{eV}$ ). As the B barrier for the PD with the InP T space, one of the following materials can be used. (1) $\operatorname{In}_{0.52} \mathrm{Al}_{0.48} \mathrm{As}$ alloy, which is isomorphic to InP. (2) $\mathrm{AlAs}_{0.54} \mathrm{Sb}_{0.46}$ alloy, which is also isomorphic to InP and forms a higher heterobarrier. (3) Since the B barrier is supposed to be very thin, it is possible to use tensile-strained $\operatorname{In}_{x} \mathrm{Al}_{1-x}$ As alloy with $x<0.52$ (up to $x=0$ ) as the barrier material. Such a barrier can be much higher and thinner than an isomorphic barrier.

As a material for the $\mathrm{C}$ contact that is much more heavily doped than the $\mathrm{T}$ space, we could use an $\mathrm{In}_{0.53} \mathrm{Ga}_{0.47} \mathrm{As}$ alloy forming the conduction band offset ${ }^{13}$ $\delta_{3}=\delta_{1}-\delta_{2} \cong 0.2 \mathrm{eV}$ (see Fig. 1) or any quaternary alloy $(\operatorname{InP})_{z}\left(\mathrm{In}_{0.53} \mathrm{Ga}_{0.47} \mathrm{As}\right)_{1-z}$ that is also isomorphic to InP. Since the C contact is heavily doped, the B barrier can be reverse biased for small values of $z$. In this case, an excessive voltage drop across this barrier occurs for a normal working regime.

It would seem that the same material as used for the $\mathrm{C}$ contact could be applied also for the A contact. But this is not the case since we need to avoid a return of reflected or backscattered high-energy electrons into the $\mathrm{T}$ space. The most effective method to mitigate such behavior is to fabricate the A contact using a multivalley semiconductor with electron $L$ and (or) $X$ valleys that have a much larger density of states than the $\Gamma$ valleys. The other method is to use a heavily doped superlattice as a material for the A contact, which will be discussed in Sec. V. To avoid quantum electron reflection, the $\mathrm{T}$ space/A contact junction should be sufficiently smoothly graded.

The maximum scattering rate ${ }^{12,14}$ for $\Gamma$ electrons in InP at $T=300 \mathrm{~K}$ and $\varepsilon<\varepsilon_{L}$ has a value $\nu \cong 1.8 \times 10^{13} \mathrm{~s}^{-1}$. This scattering is determined completely by the emission and absorption of polar optical phonons. Since such scattering is small angle and since the optical phonon energy is small in comparison to the mean electron energy (in the energy range $0-0.7 \mathrm{eV}$ ), the real electron free-path time $\tau_{\mathrm{FP}}$ must be much larger than $\nu^{-1}$. Therefore, we can write for the mean free path

$$
l_{\mathrm{FP}} \geqslant V_{S} / \nu,
$$

where $V_{S}$ is the saturated ballistic velocity, determined in a linear branch of the $\Gamma$-electron dispersion relation. The saturated ballistic velocity must be distinguished from the saturated drift velocity, which is determined by scattering processes. It is simple to check on the basis of a real dispersion relation ${ }^{12,14}$ that in most of the above-indicated energy range the electron velocity is saturated. In $\operatorname{InP}, V_{S} \cong 1.3 \times 10^{8} \mathrm{~cm} / \mathrm{s}$ and $l_{\mathrm{FP}} \geqslant\left(1.3 \times 10^{8} \mathrm{~cm} / \mathrm{s}\right) /\left(1.8 \times 10^{13} \mathrm{~s}^{-1}\right) \cong 70 \mathrm{~nm}$. The length $l_{\mathrm{FP}}$ limits the length of the ballistic $\mathrm{T}$ space where the electron velocity is determined by $V_{S}$. If $l>l_{\mathrm{FP}}$, for example, $l<2 l_{\mathrm{FP}}$, the electron transport can be considered as 
quasiballistic. Such electrons move in a sufficiently narrow beam of the $\vec{k}$ space with approximately the same quasiballistic velocity $V_{\mathrm{QB}}$, but this velocity is smaller than $V_{S}$. The small-angle character of the polar optical phonon scattering together with a comparatively high electron concentration in the $\mathrm{T}$ space promotes the development of such a picture. Electron-electron collisions result in a certain combined velocity $V_{\mathrm{QB}}$.

The other In-anion zinc blende semiconductors (InGaAs, InPSb, InAs, and InSb) which are suitable as T-space materials have parameter sets that are still more favorable than InP. They have higher energies $\varepsilon_{L}$ and $\varepsilon_{X}$, higher velocities $V_{S}$, and smaller scattering rates $v$. It is probable that $l_{\mathrm{FP}}$ $>100 \mathrm{~nm}$ can be reached in them. These materials have narrow band gaps, but we do not fear an avalanche breakdown since the T-space length $l$ can always be selected shorter than the dead space size for the considered voltages. The unsolved problem for these T-space materials is the material selection for the A contact. This problem is one of the central problems for the proposed devices and needs separate consideration.

\section{THEORETICAL MODEL FOR ELECTRON TRANSPORT IN THE T SPACE}

The proposed model is based on calculations of electron concentration $n(x)$ and electron momentum $p(x)$ in the $\mathrm{T}$ space $(0<x<l)$. We assume that a B-barrier tunnel electron injector at $x=0$ emits an electron beam with a small momentum and energy spread (compared to the momentum and the energy obtained as a result of electron transport across the $\mathrm{T}$ space). Therefore, we can consider this beam as monodirectional and monokinetic. Together with $n(x)$ and $p(x)$, we calculate an electric field $E(x)$. The right boundary of the T space $(x=l)$ is assumed to be nonreflecting and nonscattering. Concerning the left boundary $(x=0)$, we assume that (1) the tunnel electron injector emits electrons with a small initial momentum $p(0) \ll p(l)$ and we can neglect this value, $p(0) \cong 0$, and (2) this tunnel injector has been designed so that the electric field at the left boundary of the T space, $E_{0}=E(0)$, completely defines the emitted electron current density:

$$
j=j_{0}\left(E_{0}\right) .
$$

In order to satisfy Eq. (2), we actually need the voltage drop across the $\mathrm{B}$ barrier, $U_{B}$, to be negligible in comparison with the voltage drop across the T space, $U_{T} \cong U$, where $U$ is the total voltage across the device:

$$
U_{B} \ll U_{T} \cong U .
$$

Equation (2) can be inverted as

$$
E_{0}=E(x=0)=E(j),
$$

and this dependence is actually independent of the $U j$ characteristic

$$
U=U(j) .
$$

Equation (4) $[$ or (2)] together with the condition $p(0) \cong 0$ serve as boundary conditions for the equations that determine $n(x), p(x)$, and $E(x)$ in the $\mathrm{T}$ space. These equations have the traditional quasiclassical form:

$$
j=e V(p) n,
$$

the Newton law

$$
\frac{\partial p}{\partial t}+V(p) \frac{\partial p}{\partial x}=e E,
$$

the electron current continuity equation

$$
\frac{\partial j}{\partial x}+e \frac{\partial n}{\partial t}=0,
$$

the Poisson equation

$$
\kappa_{D} \frac{\partial E}{\partial x}=e n,
$$

where $\kappa_{D}$ is the dielectric constant in the $\mathrm{T}$ space, and the electron dispersion relation (in the $\Gamma$ valley)

$$
\varepsilon=\varepsilon(p),
$$

from which we obtain the electron group velocity $V(p)$ $=d \varepsilon(p) / d p$ that appears in Eqs. (6) and (7). We have assumed in Eq. (9) that the T space does not contain any ionized impurity concentration; that is, this concentration is actually small in comparison with $n(x)$. In the stationary case $(\partial / \partial t=0)$, we have $j=$ const, and Eqs. (6) $-(10)$ can be reduced to the equation

$$
\kappa_{D} V(p) \frac{d^{2} \varepsilon(p)}{d x^{2}}=e j .
$$

Multiplying both sides of Eq. (11) by $d \varepsilon(p) / d x$ and taking into account that $d \varepsilon(p) / d x=V(p)(d p / d x)=e E$, we obtain as a result of integration on $p$ from 0 to $p(e U)$

$$
\left(2 e j / \kappa_{D}\right)^{1 / 2} l=\int_{0}^{p(e U)} \frac{V(p) d p}{\left[p+p_{0}(j)\right]^{1 / 2}} .
$$

In Eq. (12), $p(e U)$ is the electron momentum at $x=l$ [where $\varepsilon(p) \cong e U]$ and $p_{0}(j)=e^{2} E^{2}(j) \kappa_{D} / 2 e j$. Equation (12) gives us the stationary $U j$ characteristic of the $\mathrm{T}$ space with the boundary conditions Eq. (4) and $p(0) \cong 0$ at $x=0$ and for a nonreflecting boundary at $x=l$.

Let us select in Eq. (12) an isotropic form of the $\Gamma$-electron dispersion relation:

$$
\varepsilon(p)=V_{S}\left(\sqrt{p_{S}^{2}+p^{2}}-p_{S}\right),
$$

where $V_{S}$ is the saturated $\Gamma$-electron velocity, $p_{S}=m V_{S}$, and $m$ is the effective mass at $p \rightarrow 0$. This selection allows us to transfer from the parabolic dispersion relation

$$
\varepsilon(p)=p^{2} / 2 m
$$

justified for small momentum values $\left(p^{2} \ll m^{2} V_{S}^{2}\right)$ to the linear law

$$
\varepsilon(p)=V_{S}\left(p-m V_{S}\right)
$$

for $p^{2} \gg m^{2} V_{S}^{2}$.

Assuming that the distribution of ballistic or quasiballistic electrons in the $\mathrm{T}$ space is localized around some average electron, we can write

$$
\varepsilon(p)=e U(x)
$$


with $p=p(x)$ where $U(x)$ is the potential in the plane $x$ of the $\mathrm{T}$ space relating to the $\mathrm{B}$ barrier, and $p(x)$ is the average electron momentum in this plane. Then we can write

$$
\begin{aligned}
\varepsilon(u) & =m V_{S}^{2} u, p(u)=m V_{S} \sqrt{u(u+2)}, V(u) \\
& =V_{S} \frac{\sqrt{u(u+2)}}{u+1}, m^{-1}(u)=m^{-1}(u+1)^{-3},
\end{aligned}
$$

and

$$
x(u)=\tau_{S} V_{S} \int_{0}^{u} \frac{d u}{\left[u^{1 / 2}(u+2)^{1 / 2}+A_{0}^{2}\right]^{1 / 2}},
$$

with

$$
A_{0}=\frac{e E(j) \tau_{S}}{m V_{S}}
$$

and

$$
\tau_{S}=\left(\frac{\kappa_{D} m V_{S}}{2 e j}\right)^{1 / 2}
$$

Since the dependence $j=j_{0}\left(E_{0}\right)$ in Eq. (2) describes an exponentially sharp increase in $j$ with an increase in $E_{0}$ $=E(0)$ for the actual area of values of $j$ and $E$, the behavior of the function $A_{0}=A_{0}(j)$ is defined mainly not by the slowly increasing inverted function $E(j)$ but by the function $\tau_{S}(j)$ from Eq. (19b). Realistic actual values of $A_{0}$ are around 1-3 and do not usually exceed 10 (see below in Sec. IV C).

For a particular value of current density $j$ a plane $x(u)$ in the $\mathrm{T}$ space can be characterized by a dimensionless transit time $\theta(u)$ along with a dimensionless potential $u(x)$ :

$$
\begin{aligned}
\theta(u) & =\tau_{S}^{-1} \int_{0}^{x(u)} d x^{\prime} / V\left(x^{\prime}\right)=\int_{0}^{u} d u^{\prime} / B\left(u^{\prime}\right) \\
& =2\left\{\left[u^{1 / 2}(u+2)^{1 / 2}+A_{0}^{2}\right]^{1 / 2}-A_{0}\right\}
\end{aligned}
$$

with $B(u)=u^{1 / 2}(u+2)^{1 / 2}\left[u^{1 / 2}(u+2)^{1 / 2}+A_{0}^{2}\right]^{1 / 2} /(u+1)$.

Considering the form of the $U j$ characteristic defined by Eq. (12) for the selected dispersion relation (13) yields

$$
l=\tau_{S} V_{S} \int_{0}^{u_{l}} \frac{d u}{\left[u^{1 / 2}(u+2)^{1 / 2}+A_{0}^{2}\right]^{1 / 2}},
$$

where $u_{l}=e U(l) / m V_{S}^{2}$. If $A_{0}^{2}=0$ and $e U(l) \ll m V_{S}^{2}$, Eq. (21) leads to

$$
j=(8 / 9)\left(\kappa_{D} / l^{2}\right)(e / 2 m)^{1 / 2} U^{3 / 2}(l) .
$$

This is the well-known Child-Langmuir law corresponding to a parabolic electron dispersion relation and a space-charge limited current $\left(A_{0}^{2}=0\right)$. If, as above, $A_{0}^{2}=0$ but $u_{l}$ $=e U(l) / m V_{S}^{2}$ in Eq. (21) is arbitrarily large, the $U j$ characteristic is described by the formula

$$
j / j_{S}=\left(\int_{0}^{u_{l}} d u / \sqrt[4]{u(u+2)}\right)^{2}
$$

with an elliptic integral on the right hand side and $j_{S}$ $=\kappa_{D} m V_{S}^{3} / 2 e l^{2}$. The dependence $j / j_{S}=F\left(u_{l}\right)$ presented by Eq. (23) is shown in Fig. 2, where we can also see the de-

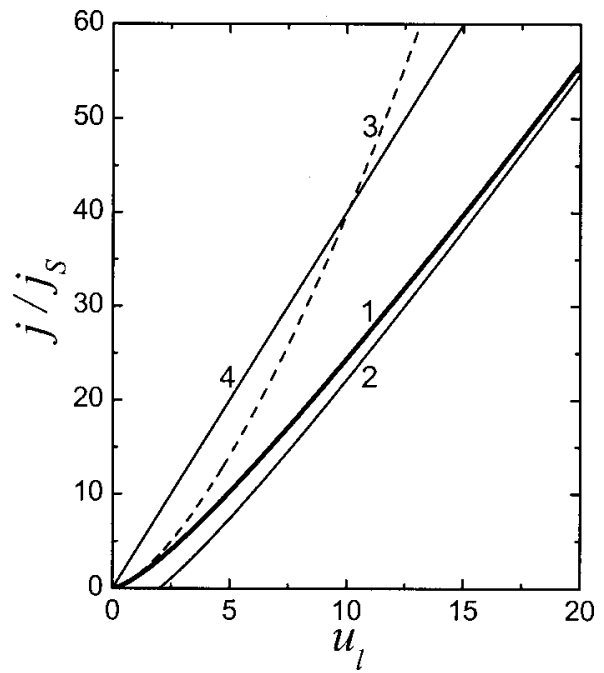

FIG. 2. dc $U j$ characteristics $j / j_{S}=F\left(u_{l}\right)$ for $\mathrm{T}$ space in the case of the space-charge limited current presented by Eq. (23) (curve 1), and by approximate formulas $j / j_{S}=4\left(u_{l}-\sqrt{2 u_{l}}\right)$ (curve 2), $j / j_{S}=(8 / 9) \sqrt{2} u_{l}^{3 / 2}$ (curve 3 ), and $j / j_{S}=4 u_{l}$ (curve 4 ).

pendence $j / j_{S}=(8 \sqrt{2}) u_{l}^{3 / 2} / 9$ [that is, Eq. (22) coinciding with Eq. (23) for $\left.u_{l} \leqslant 1\right]$ and the dependence $j / j_{S}=4\left(u_{l}\right.$ $-\sqrt{2 u_{l}}$ ) nearing Eq. (23) for very large $u_{l}$. As is seen, the last dependence is very far from the linear dependence presented by only the first component in the parentheses. A substantial deviation from the results of Eq. (21) occurs if $u_{l}$ $>2.5$, which is unreachable in the structures considered.

At large values of $A_{0}$ (when $A_{0}^{2}>1$ ), Eq. (21) leads to the approximate formula

$$
U(l)=E(j) l+\left(j l^{2} / 2 \kappa_{D} V_{S}\right) .
$$

The first component on the right hand side of Eq. (24) corresponds to a homogeneous electric field $E(x)=E(j)$ in the whole $\mathrm{T}$ space. The second component takes into account the contribution of the electron space charge. The role of this charge increases with an increase in the length $l$ of the T space.

In the general case, the calculation of the $U j$ characteristic from Eq. (21) requires detailed knowledge of $E(j)$ in Eq. (19a). Calculation of such functions for specific B barriers is very important but is not addressed in this article.

\section{HIGH-FREQUENCY ADMITTANCE OF THE T SPACE (SMALL-SIGNAL APPROXIMATION)}

\section{A. General formulas}

A small-signal high-frequency admittance can be calculated after transition from the stationary values $j, p$, and $E$ to the sums $j \rightarrow j+j^{\prime}(x) \exp (i \omega t), \quad p \rightarrow p+p^{\prime}(x) \exp (i \omega t), \quad E$ $\rightarrow E+E^{\prime} \exp (i \omega t)$, where $\omega$ is the frequency of a small harmonic signal, and the amplitudes $j^{\prime}(x), p^{\prime}(x), E^{\prime}(x)$, etc. satisfy the system of equations 


$$
\begin{aligned}
& j^{\prime}=e V n^{\prime}+e n m^{-1}(p) p^{\prime}, \\
& \kappa_{D} \frac{d E^{\prime}}{d x}=e n^{\prime}, \\
& \frac{d j^{\prime}}{d x}+i \omega e n^{\prime}=0, \\
& i \omega p^{\prime}+V \frac{d p^{\prime}}{d x}+m^{-1}(p) p^{\prime} \frac{d p}{d x}=e E^{\prime},
\end{aligned}
$$

where $V=V(p)=d \varepsilon(p) / d p$ and $m^{-1}(p)=d^{2} \varepsilon(p) / d p^{2}$ as above. We can see from Eqs. (26) and (27) that instead of the electron current density $j^{\prime}(x)$ only the total current density

$$
J^{\prime}=j^{\prime}(x)+i \omega \kappa_{D} E^{\prime}(x)
$$

is constant thoughout the $\mathrm{T}$ space. By taking this condition, into account we obtain an equation that determines $p^{\prime}(x)$ :

$$
\begin{aligned}
\left(V \frac{d}{d x}+i \omega\right)\left(V \frac{d}{d x}+i \omega+m^{-1}(p) \frac{d p}{d x}\right) p^{\prime}+\frac{e^{2} n}{\kappa_{D}} m^{-1}(p) p^{\prime} \\
=\frac{e J^{\prime}}{\kappa_{D}} \equiv \lambda .
\end{aligned}
$$

This equation should be solved with boundary conditions at $x=0$; they are

$$
p^{\prime}(0)=0
$$

and

$$
j^{\prime}(0)=\sigma_{S} E^{\prime}(0),
$$

where we introduce the "conductivity" of the tunnel emitter: $\sigma_{S}=d j /\left.d E\right|_{x=0}$. The boundary condition (31b) can be rewritten in the form

$$
J^{\prime}=\left(\sigma_{S}+i \omega \kappa_{D}\right) E^{\prime}(0) .
$$

Replacing in Eq. (30) the independent variable $x$ with the new variable $u=u(x)$, we can present this equation in the form of two equations of the first degree:

$$
\begin{aligned}
& {\left[B(u) \frac{d}{d u}+i \Omega\right] \beta-\alpha=0,} \\
& {\left[B(u) \frac{d}{d u}+i \Omega\right] \alpha+Y_{1}(u) \alpha-Y_{2}(u) \beta-1=0,}
\end{aligned}
$$

where $\beta=p^{\prime} / \lambda \tau_{S}^{2}, \alpha=[B(u) d / d u+i \Omega] \beta, \Omega=\omega \tau_{S}, B(u)$ is defined at Eq. (20),

$$
Y_{1}(u)=\left[u^{1 / 2}(u+2)^{1 / 2}+A_{0}^{2}\right]^{1 / 2} / u^{1 / 2}(u+2)^{1 / 2}(u+1)^{2},
$$

and

$Y_{2}(u)=\frac{2 \sqrt{u(u+2)}}{(u+1)^{4}}+A_{0}^{2}\left[\frac{2}{(u+1)^{4}}+\frac{1}{u(u+2)(u+1)^{2}}\right]$.

A simpler and more convenient form of Eqs. (33) and (34) appears as a result of transfer to the variable $\theta(u)$ [see Eq. (20)]:

$$
\begin{aligned}
& \left(\frac{d}{d \theta}+i \Omega\right) \beta-\alpha=0, \\
& \left(\frac{d}{d \theta}+i \Omega\right) \alpha+S_{1}(\theta) \alpha-S_{2}(\theta) \beta-1=0,
\end{aligned}
$$

where

$$
\begin{aligned}
& S_{1}(\theta)=Y_{1}(u)=\frac{A_{0}+\theta / 2}{\theta\left(A_{0}+\theta / 4\right)\left[1+\theta^{2}\left(A_{0}+\theta / 4\right)^{2}\right]}, \\
& S_{2}(\theta)=Y_{2}(u)=\frac{A_{0}^{2}+3 A_{0}^{2} \theta^{2}\left(A_{0}+\theta / 4\right)^{2}+2 \theta^{3}\left(A_{0}+\theta / 4\right)^{3}}{\theta^{2}\left(A_{0}+\theta / 4\right)^{2}\left[1+\theta^{2}\left(A_{0}+\theta / 4\right)^{2}\right]^{2}} .
\end{aligned}
$$

Equations (35) and (36) [or (33) and (34)] should be solved with the boundary conditions at $\theta=0$ [or $u=0$; see Eqs. (31a) and (31b)]

$$
\begin{aligned}
& \beta(0)=0, \\
& \left.\frac{d \beta}{d \theta}\right|_{\theta=0}=\left.B(u) \frac{d \beta}{d u}\right|_{u=0}=\alpha(0)=\left(\Omega_{0}+i \Omega\right)^{-1},
\end{aligned}
$$

where $\Omega_{0}=\sigma_{S}\left(m V_{S} / 2 e j \kappa_{D}\right)^{1 / 2}$. The value of $\Omega_{0}(j)$ increases comparatively rapidly with increase in $j$ and $E$. As shown below, this increase in $\Omega_{0}(j)$ can substantially control the frequency range of negative conductance.

\section{B. Space-charge limited case}

The simplest case occurs when the tunnel emitter B barrier does not limit electron injection, and it is limited by only the electron space charge. Then $A_{0}=0$ and $\sigma_{S} \rightarrow \infty$, that is, $\left.(d \beta / d \theta)\right|_{\theta=0}=\alpha(0)=0$. In this space-charge limited case

$S_{1}(\theta)=2 / \theta\left[1+(\theta / 2)^{4}\right], S_{2}(\theta)=\theta^{2} / 2\left[1+(\theta / 2)^{4}\right]^{2}$.

The second components in the square brackets in the denominators of $S_{1,2}(\theta)$ [Eqs. (40)], which are equal to $(\theta / 2)^{4}$, are the result of nonparabolicity and should be excluded for the parabolic limit $(\theta \ll 2)$ as well as the whole small component $-S_{2}(\theta) \beta$ in Eq. (36).

In the parabolic case, the desired solution of Eqs. (35) and (36) can be presented in the form

$\beta(\theta)=-\Omega^{-2}\left[1+2 i \chi^{-1}+\left(1-2 i \chi^{-1}\right) \exp (-i \chi)\right]$,

where $\chi=\Omega \theta$. It allows us to calculate the high-frequency electric field distribution in the $\mathrm{T}$ space:

$$
\begin{aligned}
E^{\prime}(\theta)= & -\left(\lambda \tau_{S} / \Omega\right)\left[i\left(1+2 \chi^{-2}\right)+2 \chi^{-1}\left(1-i \chi^{-1}\right)\right. \\
& \times \exp (-i \chi)]
\end{aligned}
$$

and the impedance of the $\mathrm{T}$ space:

$$
\begin{aligned}
Z=R+i X= & R_{0}\left\{2\left(1-\cos \chi_{l}\right)-\chi_{l} \sin \chi_{l}-i\left[\chi_{l}\left(1-\cos \chi_{l}\right)\right.\right. \\
& \left.\left.-2 \sin \chi_{l}+\chi_{l}^{3} / 6\right]\right\},
\end{aligned}
$$

where $\chi_{l}=\Omega \theta_{l}=\omega\left(6 m \kappa_{D} l / e j\right)^{1 / 3}, \theta_{l}=\theta\left(u_{l}\right)=\theta(x=l)$, and $R_{0}=e j / \kappa_{D}^{2} m \omega^{4}$.

We can see that the active impedance component $R$ $=R_{0}\left[2\left(1-\cos \chi_{l}\right)-\chi_{l} \sin \chi_{l}\right]$ contains wide negative resistance windows for $\chi$ that are in the intervals $(2 \pi, 3 \pi)$, $(4 \pi, 5 \pi)$, and so on. Evidently, Benham ${ }^{15}$ first paid attention to this important result. There were several successful tries at exciting high-frequency oscillations in the first (the lowest frequency) of these windows. For example, oscillations with the wavelength $\sim 11.5 \mathrm{~cm}$ were generated $^{16}$ in vacuum diodes with thermionic electron emission. 

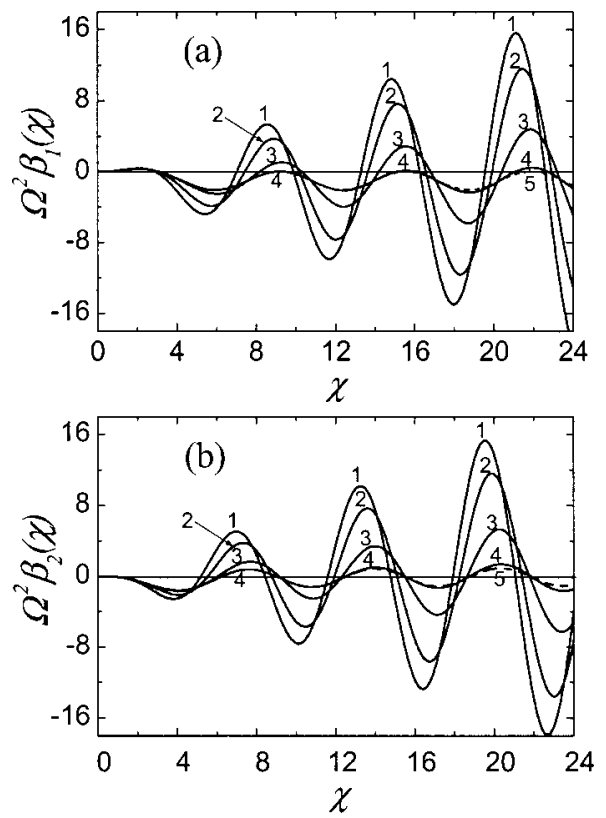

FIG. 3. Functions $\Omega^{2} \beta_{1}(\chi)$ (a) and $\Omega^{2} \beta_{2}(\chi)$ (b) for four values of the normalized frequency $\Omega=\omega \tau_{S}: \Omega=0.3 \pi$ (curve 1), $0.5 \pi$ (curve 2), $1.0 \pi$ (curve 3), $3.0 \pi$ (curve 4), and in the parabolic case when $\theta=\chi / \Omega \ll 2$ (dashed curves 5).

Note that in both the specific parabolic case and the more general nonparabolic case [Eq. (13)] when only the full expressions for $S_{1,2}(\theta)$ in Eqs. (40) are valid, our Eqs. (35) and (36) do not include any additional parameters apart from the frequency $\Omega$ since $\beta(0)=0$ and $\alpha(0)=0$. Therefore, the desired solution $\beta(\theta)$ depends only on the single parameter $\Omega$. The functions $\beta_{1}(\chi)=\operatorname{Re} \beta(\chi)$ and $\beta_{2}(\chi)=\operatorname{Im} \beta(\chi)$ multiplied by $\Omega^{2}$ for convenience and calculated as a result of the numerical solution of Eqs. (35) and (36) [with $S_{1,2}(\theta)$ from Eqs. (40)] are presented in Fig. 3 for several values of $\Omega$. In the same Fig. 3 (see the dashed curves 5), we can see also the functions $\beta_{1,2}(\chi)$ calculated according to Eq. (41) for the parabolic limit $(\theta \ll 2)$. The high-frequency current $J^{\prime}$ generates in the T space standing waves of $n^{\prime}, E^{\prime}, V^{\prime}$, and $p^{\prime}$ due to the boundary conditions at $x=u(x)=\theta(u)$ $=0$. These standing waves are not sensitive to the length $l$ of the $\mathrm{T}$ space because of the lack of both electron reflection and an electron backscattering at the boundary $\mathrm{T}$ space/A contact.

The calculated functions $\beta(\theta)$ allow us to calculate the impedance $Z$ of the $T$ space. In the most general case it is defined by the formula

$$
Z=\frac{l^{2}}{V_{S} \kappa_{D}} \frac{I_{2}\left(\Omega, \theta_{l}\right)}{I_{1}^{2}\left(\theta_{l}\right)},
$$

where

$I_{1}\left(\theta_{l} ; A_{0}\right)=\int_{0}^{\theta_{l}} \theta\left(A_{0}+\theta / 4\right) d \theta /\left[1+\theta^{2}\left(A_{0}+\theta / 4\right)^{2}\right]^{1 / 2}$,
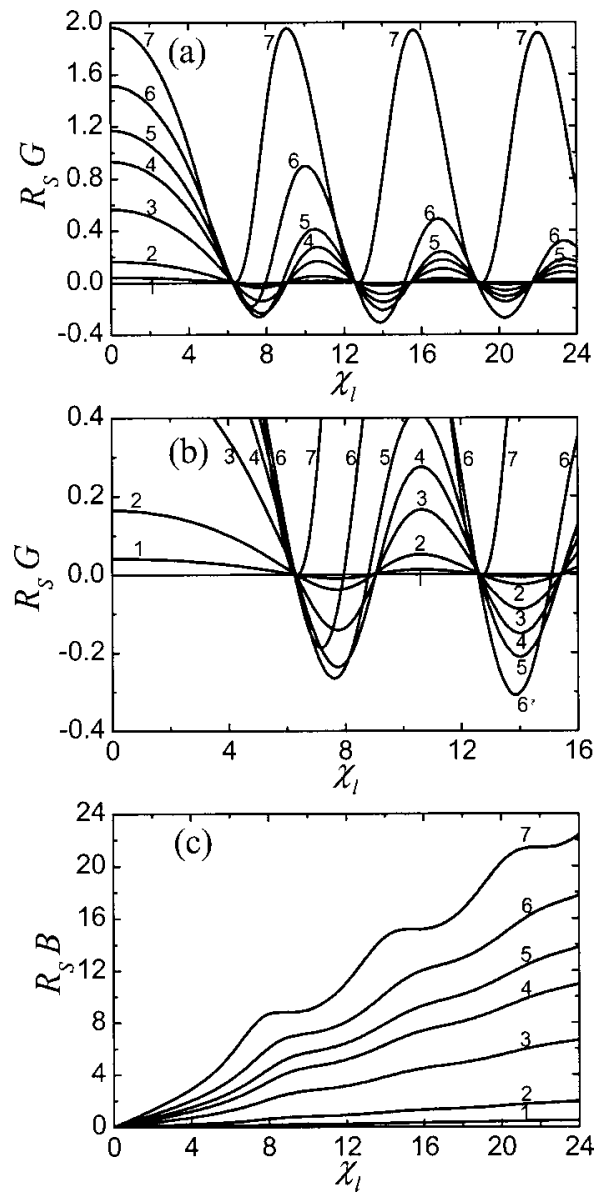

FIG. 4. Normalized conductance $R_{S} G$ (a),(b) and susceptance $R_{S} B$ (c) plotted against $\chi_{l}=\Omega \theta_{l}$ for different values of $\theta_{l}: 0.5$ (curve 1), 1.0 (curve 2), 2.0 (curve 3), 3.0 (curve 4), 4.0 (curve 5), 7.0 (curve 6), and 100.0 (curve 7). (a) and (b) differ from each other only in scale.

$$
\begin{aligned}
I_{2}\left(\Omega, \theta_{l} ; A_{0}, \Omega_{0}\right)= & \int_{0}^{\theta_{l}}\left[\alpha+\beta S_{1}(\theta)\right] \\
& \times \theta\left(A_{0}+\theta / 4\right) d \theta /\left[1+\theta^{2}\left(A_{0}+\theta / 4\right)^{2}\right]^{1 / 2} .
\end{aligned}
$$

In the case of the space-charge limited current under consideration, we should use the simplified expressions for $I_{1}\left(\theta_{l}\right)$ and $I_{2}\left(\Omega, \theta_{l}\right)$ with $A_{0}=0, \alpha(0)=0\left(\Omega_{0}=\infty\right), \beta(0)=0$, and $S_{1,2}(\theta)$ described by Eqs. (40). Note that in this case there is a very simple connection between $u_{l}$ and $\theta_{l}$ in the form $\theta_{l}$ $=2\left[u_{l}\left(u_{l}+2\right)\right]^{1 / 4}$. The calculated functions $R_{S} G\left(\chi_{l}\right)$ and $R_{S} B\left(\chi_{l}\right) \quad$ where $\quad \chi_{l}=\Omega \theta_{l}, \quad R_{S}=l^{2} / V_{S} \kappa_{D}, \quad G(\chi)$ $=(\operatorname{Re} Z) /|Z|^{2}$, and $B(\chi)=-(\operatorname{Im} Z) /|Z|^{2}$ for several different values of $\theta_{l}$ are shown in Fig. 4. Since $\chi_{l}=\Omega \theta_{l}=\omega \tau_{S} \theta_{l}$, these plots are in fact the real and imaginary components of admittance $1 / Z$ plotted against the frequency $\omega$ for different voltages $u_{l}$ across the T space. It can be seen that the product $\tau_{S} \theta_{l}$ is determined by $u_{l}$ since $\tau_{S} \theta_{l}$ $=\left(\kappa_{D} m V_{S} / 2 e j\right)^{1 / 2} 2 u_{l}^{1 / 4}\left(u_{l}+2\right)^{1 / 4}$ and $j=j\left(u_{l}\right)=j_{S} F\left(u_{l}\right)$ is presented in Fig. 2. For small values of $u_{l}\left(u_{l} \ll 2\right)$ when $F\left(u_{l}\right)=(8 \sqrt{2}) u_{l}^{3 / 2} / 9$, we can obtain $\tau_{S} \theta_{l} \cong 3 l / V_{s}\left(2 u_{l}\right)^{1 / 2}$, that is, $\tau_{S} \theta_{l}$ increases with a decrease in $u_{l}$. For $u_{l} \gg 2$, we obtain $\tau_{S} \theta_{l} \cong l / V_{S}$, and $\chi_{l}=\Omega \theta_{l}=\omega \tau_{S} \theta_{l}$ is independent of the voltage. 
We can observe in Fig. 4 the above-mentioned negative conductance windows, which are maximally wide for small values of $\theta_{l}\left(\theta_{l}<2\right)$ and narrow with an increase in $\theta_{l}$ for large values of $\theta_{l}$. Their narrowing and subsequent practical disappearance is a result of the nonparabolicity of the electron dispersion relation for $\theta \geqslant 2$. This is the evident disadvantage for ballistic transit-time oscillators with spacecharge limited current.

\section{Tunnel emitter control case: Specific description}

As we know, the finite resistance of a tunnel emitter controlled by an electric field $E(x=0)=E(j)$ introduces two additional parameters $A_{0}$ and $\Omega_{0}$. Specifically, parameter $A_{0}$ substantially complicates Eqs. (37a) and (37b), which determine the functions $S_{1,2}(\theta)$. These formulas can be noticeably simplified in the parabolic limit if, along with a strong inequality $\theta \ll 2$, an additional condition is satisfied: $\theta$ $\ll 2 A_{0}$. In this case, Eqs. (37a) and (37b) lead to

$$
\begin{aligned}
& S_{1}(\theta) \cong 1 / \theta, \\
& S_{2}(\theta) \cong \frac{1}{\theta^{2}}+2 A_{0}^{2} .
\end{aligned}
$$

Substitution of expressions (47a) and (47b) into Eqs. (35) and (36) leads to a differential equation for the function $\beta_{0}(\theta)=\beta(\theta) \exp (i \Omega \theta)$ in the form

$$
\begin{aligned}
& \theta^{2}\left(d^{2} \beta_{0} / d \theta^{2}\right)+\theta\left(d \beta_{0} / d \theta\right)-\left(2 A_{0}^{2} \theta^{2}+1\right) \beta_{0} \\
& \quad=\theta^{2} \exp (i \Omega \theta),
\end{aligned}
$$

which is an inhomogeneous modified Bessel equation. ${ }^{17}$ The fundamental solution of Eq. (48) is

$$
\begin{aligned}
\beta_{0}(\theta)= & C K_{1}\left(2^{1 / 2} A_{0} \theta\right)+D I_{1}\left(2^{1 / 2} A_{0} \theta\right) \\
& +\int_{0}^{\theta} \theta^{\prime} d \theta^{\prime} \exp \left(i \Omega \theta^{\prime}\right) \\
& \times\left[K_{1}\left(2^{1 / 2} A_{0} \theta^{\prime}\right) I_{1}\left(2^{1 / 2} A_{0} \theta\right)\right. \\
& \left.-K_{1}\left(2^{1 / 2} A_{0} \theta\right) I_{1}\left(2^{1 / 2} A_{0} \theta^{\prime}\right)\right],
\end{aligned}
$$

where $I_{1}(x)$ and $K_{1}(x)$ are the modified Bessel functions. ${ }^{17}$ Using the boundary conditions (38) and (39), we can obtain $C=0, D=2^{1 / 2} / A_{0}\left(\Omega_{0}+i \Omega\right)$, and

$$
\begin{aligned}
\beta(\theta)= & {\left[2^{1 / 2} / A_{0}\left(\Omega_{0}+i \Omega\right)\right] \exp (-i \Omega \theta) I_{1}\left(2^{1 / 2} A_{0} \theta\right) } \\
& +\int_{0}^{\theta} \theta^{\prime} d \theta^{\prime} \exp \left[i \Omega\left(\theta^{\prime}-\theta\right)\right] \\
& \times\left[K_{1}\left(2^{1 / 2} A_{0} \theta^{\prime}\right) I_{1}\left(2^{1 / 2} A_{0} \theta\right)\right. \\
& \left.-K_{1}\left(2^{1 / 2} A_{0} \theta\right) I_{1}\left(2^{1 / 2} A_{0} \theta^{\prime}\right)\right] .
\end{aligned}
$$

The impedance of the $\mathrm{T}$ space is determined as above by Eq. (44), but in the case under consideration $\theta \ll 2$ and $2 A_{0}$ we can substitute $\alpha+\beta S_{1}=d \beta / d \theta+i \Omega \beta+\beta / \theta$ and substantially simplify the integrands in Eqs. (45) and (46): $\theta\left(A_{0}\right.$ $+\theta / 4) /\left[1+\theta^{2}\left(A_{0}+\theta / 4\right)^{2}\right]^{1 / 2} \cong A_{0} \theta /\left(1+A_{0}^{2} \theta^{2}\right)^{1 / 2}$.

Equation (50) demonstrates that $\beta(\theta)$ can be presented in the form of the sum of two components:

$$
\beta(\theta)=\beta^{(1)}(\theta)+\beta^{(2)}(\theta),
$$

where $\beta^{(1)}(\theta)$ is the second component on the right hand side of Eq. (50) and $\beta^{(2)}(\theta)$ is the first one. The function $\beta^{(1)}(\theta)$ is the solution of Eqs. (35) and (36) for $\alpha(0)$ $=\beta(0)=0$ and $\beta^{(2)}(\theta)$ is the additional component introduced by a finite value of $\alpha(0)=d \beta /\left.d \theta\right|_{\theta=0}=1 /\left(\Omega_{0}\right.$ $+i \Omega)$. It is convenient to write

$$
\beta^{(2)}(\theta)=\widehat{\beta}^{(2)}(\theta) /\left(\Omega_{0}+i \Omega\right),
$$

where $\widehat{\beta}^{(2)}(\theta)$ is the solution of the same Eqs. (35) and (36) but without the component -1 on the left hand side of Eq. (36) and for the boundary conditions $\widehat{\beta}^{(2)}(0)=0$ and $\widehat{\alpha}^{(2)}(0)=d \widehat{\beta}^{(2)} /\left.d \theta\right|_{\theta=0}=1$.

\section{Tunnel emitter control case: General description}

It is not difficult to make sure that the solution structure in the form of Eqs. (51) and (52) occurs in the most general case for arbitrary values of $\theta, \theta_{l}$, and $A_{0}$. Therefore, in the general case, the impedance $Z$ can be expressed as the sum of two components

$$
Z=Z^{(1)}\left(\Omega, \theta_{l} ; A_{0}\right)+\left(\Omega_{0}+i \Omega\right)^{-1} \hat{Z}^{(2)}\left(\Omega, \theta_{l} ; A_{0}\right) .
$$

The impedance $Z^{(1)}=R^{(1)}+i X^{(1)}$ is generated by the component $\beta^{(1)}(\theta)$ :

$$
Z^{(1)}=\frac{l^{2}}{V_{S} \kappa_{D}} \frac{\int_{0}^{\theta_{l}}\left[\left(d \beta^{(1)} / d \theta\right)+i \Omega \beta^{(1)}+\beta^{(1)} S_{1}(\theta)\right] \theta\left(A_{0}+\theta / 4\right) d \theta /\left[1+\theta^{2}\left(A_{0}+\theta / 4\right)^{2}\right]^{1 / 2}}{I_{1}^{2}\left(\theta_{l} ; A_{0}\right)},
$$

and the impedance $\hat{Z}^{(2)}=\hat{R}^{(2)}+i \hat{X}^{(2)}$ is generated by $\hat{\beta}^{(2)}(\theta)$ : to obtain the appropriate formula, we should replace $\beta^{(1)}(\theta)$ in the integrand in Eq. (54) by $\hat{\beta}^{(2)}(\theta)$. Then, the real component $R$ and imaginary component $X$ of the total impedance $Z=R$ $+i X$ are determined by the following formulas: 


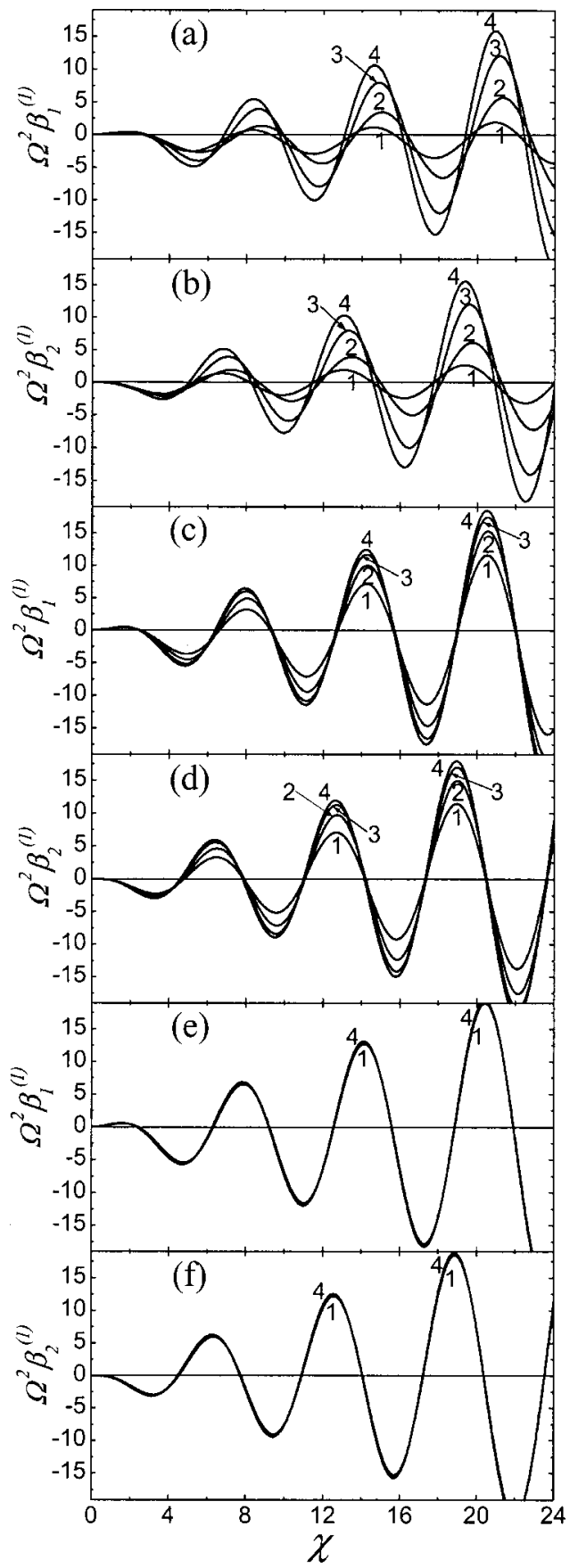

FIG. 5. Functions $\Omega^{2} \beta_{1}^{(1)}(\chi)[(\mathrm{a}),(\mathrm{c}),(\mathrm{e})]$ and $\Omega^{2} \beta_{2}^{(1)}(\chi)[(\mathrm{b}),(\mathrm{d}),(\mathrm{f})]$ for four values of the normalized frequency $\Omega=\omega \tau_{S}: \Omega=0.3 \pi$ (curve 1), $0.5 \pi$ (curve 2), $1.0 \pi$ (curve 3 ), $3.0 \pi$ (curve 4 ), and for three values of parameter $A_{0}: A_{0}=0.1$ (a),(b), $1.0(\mathrm{c}),(\mathrm{d})$, and $10.0(\mathrm{e}),(\mathrm{f})$.

$$
\begin{aligned}
& R=R^{(1)}+\left(\Omega_{0} \hat{R}^{(2)}+\Omega \hat{X}^{(2)}\right) /\left(\Omega_{0}^{2}+\Omega^{2}\right), \\
& X=X^{(1)}+\left(\Omega_{0} \hat{X}^{(2)}-\Omega \hat{R}^{(2)}\right) /\left(\Omega_{0}^{2}+\Omega^{2}\right) .
\end{aligned}
$$

The calculated $Z$ components in Eqs. (55a) and (55b) allow us to calculate the components of the admittance $G\left(\chi_{l}\right)$ $=(\operatorname{Re} Z) /|Z|^{2}$ and $B\left(\chi_{l}\right)=-(\operatorname{Im} Z) /|Z|^{2}$.

The real and imaginary components of the function $\beta^{(1)}(\theta)$ are shown in Fig. 5 for $A_{0}=0.1,1$, and 10 and for the same values of $\Omega$ as in Fig. 3 for $A_{0}=0$. We can see an evident tendency of the curves to merge with one another at $A_{0}=1$ and become the same at $A_{0}=10$. The latter is con-

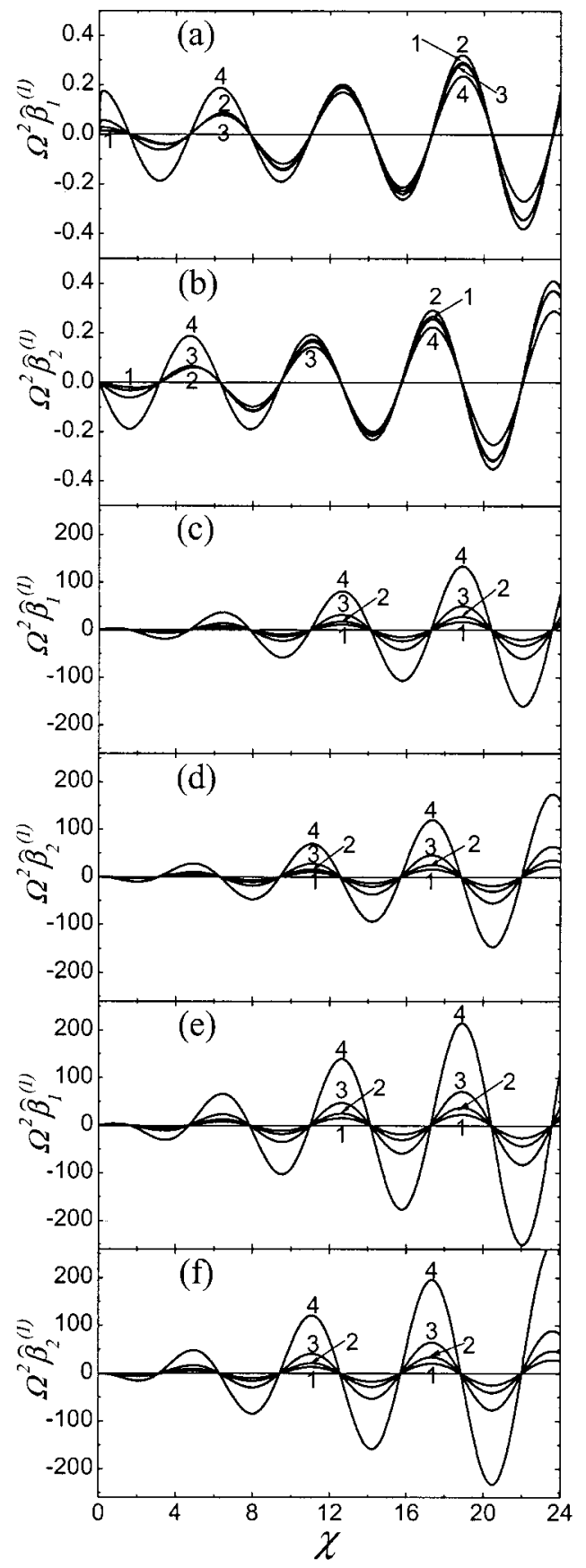

FIG. 6. Functions $\Omega^{2} \hat{\beta}_{1}^{(2)}(\chi)[(\mathrm{a}),(\mathrm{c}),(\mathrm{e})]$ and $\Omega^{2} \hat{\beta}_{2}^{(2)}(\chi)[(\mathrm{b}),(\mathrm{d}),(\mathrm{f})]$ for four values of the normalized frequency $\Omega=\omega \tau_{S}: \Omega=0.3 \pi$ (curve 1), $0.5 \pi$ (curve 2), $1.0 \pi$ (curve 3), $3.0 \pi$ (curve 4 ), and for three values of parameter $A_{0}: A_{0}=0$ (a),(b), $A_{0}=1.0$ (c),(d), and $A_{0}=10.0$ (e),(f).

nected with the decreasing coefficients $S_{1}(\theta)$ and $S_{2}(\theta)$ in Eq. (36) as a result of an increase in $A_{0}$. Equations (35) and (36) almost completely lose dependence on $A_{0}$ and can be reduced to the equation

$$
(d / d \theta+i \Omega)^{2} \beta-1=0 .
$$

This equation has for $\alpha(0)=d \beta /\left.d \theta\right|_{\theta=0}=0$ and $\beta(0)=0$ the solution $\beta(\chi)=\beta_{1}(\chi)+i \beta_{2}(\chi)$ with $\beta_{1} \Omega^{2}=-(1$ $-\cos \chi-\chi \sin \chi)$ and $\beta_{2} \Omega^{2}=-(\sin \chi-\chi \cos \chi)$, which is in good agreement with the curves in Fig. 5(c). As above, $\chi$ $=\Omega \theta$. 

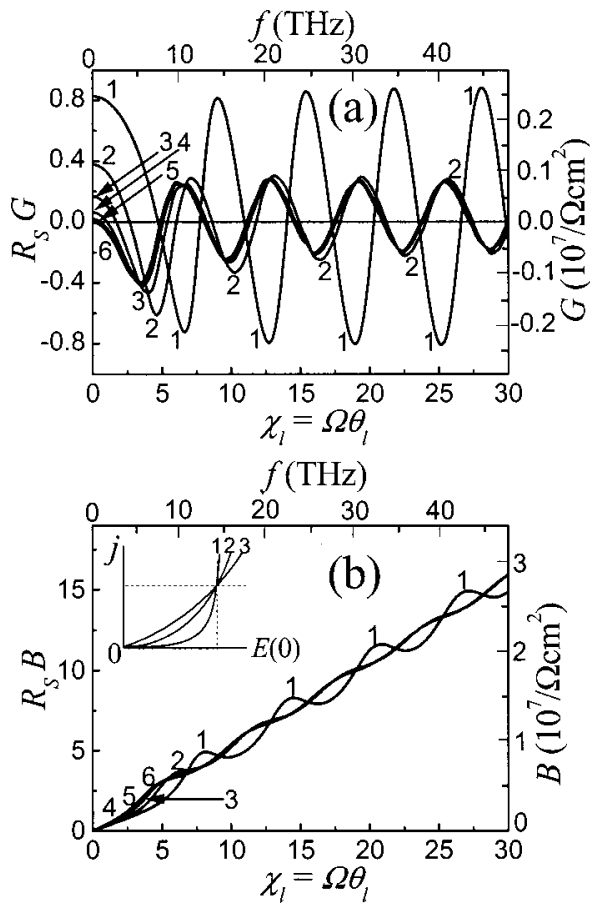

FIG. 7. Conductance $G$ (a) and susceptance $B$ (b) plotted against frequency $f$ for different values of $\Omega_{0}\left[\Omega_{0}=\infty\right.$ (curve 1), 10.0 (curve 2), 3.0 (curve 3), 1.0 (curve 4), 0.3 (curve 5), and 0 (curve 6)] at the current density $j$ $=10^{4} \mathrm{~A} / \mathrm{cm}^{2}$. The inset in (b) demonstrates the appearance of different values of $\Omega_{0}$ at the same value of $A_{0}$.

The real and imaginary components of the functions $\hat{\beta}^{(2)}(\theta)$ are shown in Fig. 6 for the same values of the normalized frequency $\Omega$ and for $A_{0}=0,1.0$, and 10 . Contrary to the functions $\Omega^{2} \beta^{(1)}(\theta)$, the functions $\Omega^{2} \hat{\beta}^{(2)}(\theta)$ do not
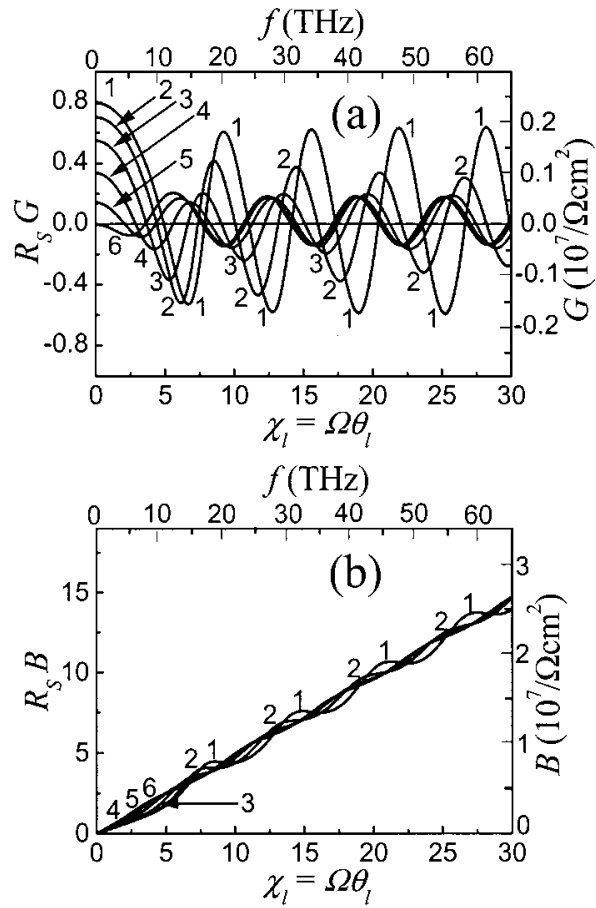

FIG. 8. Conductance $G$ (a) and susceptance $B$ (b) plotted against frequency $f$ for different values of $\Omega_{0}\left[\Omega_{0}=\infty\right.$ (curve 1), 10.0 (curve 2), 3.0 (curve 3), 1.0 (curve 4), 0.3 (curve 5), and 0 (curve 6)] at the current density $j$ $=10^{6} \mathrm{~A} / \mathrm{cm}^{2}$.

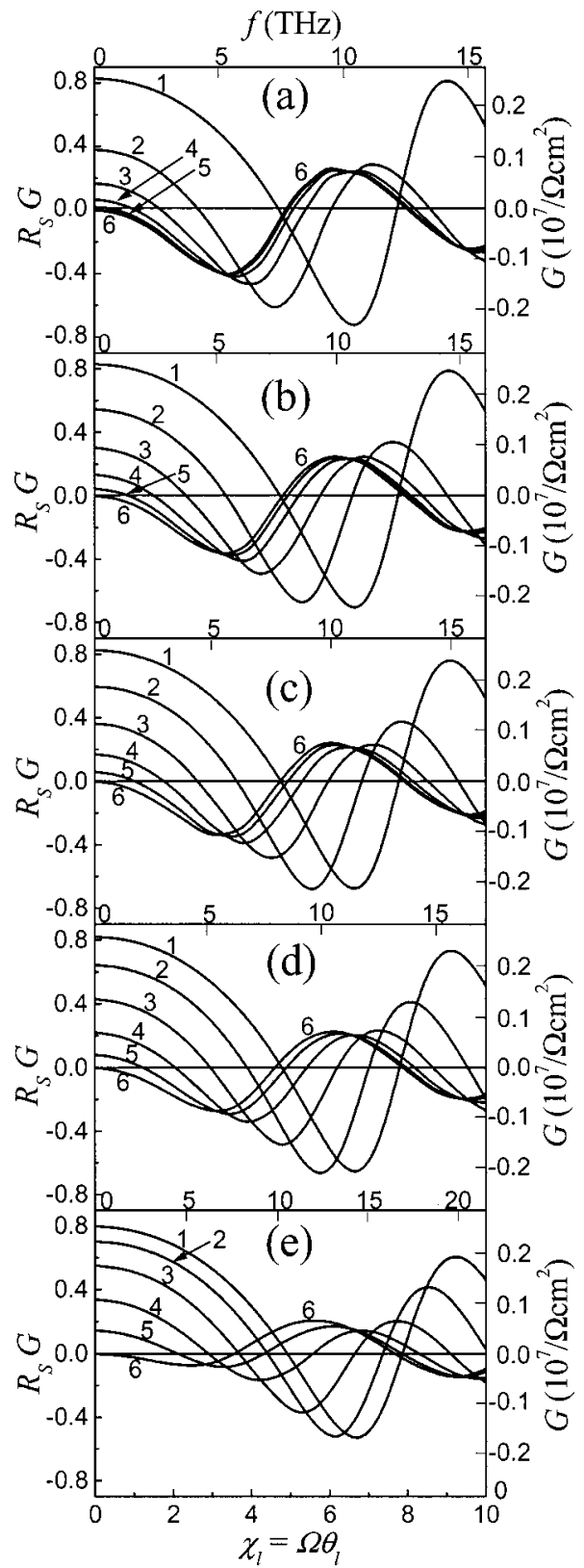

FIG. 9. Conductance $G$ plotted against frequency $f$ for different values of $\Omega_{0}\left[\Omega_{0}=\infty\right.$ (curve 1), 10.0 (curve 2), 3.0 (curve 3), 1.0 (curve 4), 0.3 (curve 5), and 0 (curve 6)] at the current density $j=10^{4} \mathrm{~A} / \mathrm{cm}^{2}$ (a), 5 $\times 10^{4} \mathrm{~A} / \mathrm{cm}^{2}$ (b), $10^{5} \mathrm{~A} / \mathrm{cm}^{2}$ (c), $2 \times 10^{5} \mathrm{~A} / \mathrm{cm}^{2}$ (d), and $10^{6} \mathrm{~A} / \mathrm{cm}^{2}$ (e).

become closer to each other for large $A_{0}$; some of them even diverge. Such behavior is connected with an effect of the boundary condition $\widehat{\alpha}^{(2)}(0)=d \hat{\beta}^{(2)} /\left.d \theta\right|_{\theta=0}=1$. The solutions of the equation $(d / d \theta+i \Omega)^{2} \beta=0$ with this boundary condition are $\beta_{1} \Omega^{2}=\Omega \chi \cos \chi$ and $\beta_{2} \Omega^{2}=-\Omega \chi \sin \chi$. They are proportional to $\Omega$.

In Figs. 7-10, we compare the real and imaginary admittance components $G\left(\chi_{l}\right)$ and $B\left(\chi_{l}\right), \chi_{l}=\Omega \theta_{l}$, for several sets of parameters of the $T$ space and the tunnel emitter. All of these parameters are chosen for the single selected $\mathrm{T}$ space with length $l=0.7 \times 10^{-5} \mathrm{~cm}=70 \mathrm{~nm}$ and with material parameters that are appropriate to InP: $m=0.75$ $\times 10^{-28} \mathrm{~g}, \kappa_{D}=12.4 / 4 \pi \cong 1, V_{S}=1.3 \times 10^{8} \mathrm{~cm} / \mathrm{s}, R_{S}=3.46$ $\times 10^{-7} \Omega \mathrm{cm}^{2}$, and $U(l)=0.6 \mathrm{~V}$. The value of $V_{S}$ is selected 


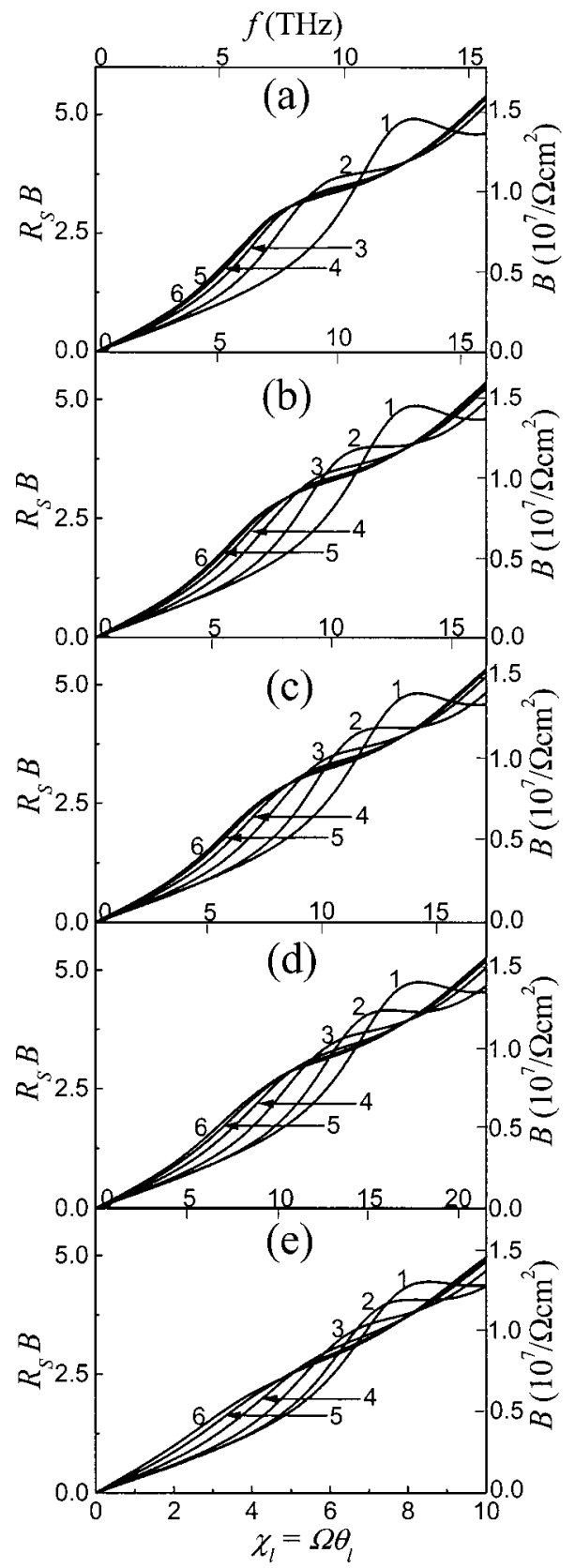

FIG. 10. Susceptance $B$ plotted against frequency $f$ for different values of $\Omega_{0}\left[\Omega_{0}=\infty\right.$ (curve 1), 10.0 (curve 2), 3.0 (curve 3), 1.0 (curve 4), 0.3 (curve 5 ), and 0 (curve 6)] at the current density $j=10^{4} \mathrm{~A} / \mathrm{cm}^{2}$ (a), 5 $\times 10^{4} \mathrm{~A} / \mathrm{cm}^{2}$ (b), $10^{5} \mathrm{~A} / \mathrm{cm}^{2}$ (c), $2 \times 10^{5} \mathrm{~A} / \mathrm{cm}^{2}$ (d), and $10^{6} \mathrm{~A} / \mathrm{cm}^{2}$ (e).

in accordance with the data extracted from Ref. 12. The value of $e U(l)$ is chosen to be equal to the $L$-valley bottom energy in InP.

This set of parameters allows us to calculate the normalized value of the voltage across the $\mathrm{T}$ space, $u_{l}$ $=e U(l) / m V_{S}^{2}=0.76$, the average value of the electric field in the T space, $\bar{E}=0.86 \times 10^{5} \mathrm{~V} / \mathrm{cm}$, the characteristic current density in Eq. (23), $j_{S}=\kappa_{D} m V_{S}^{3} / 2 e l^{2}=1.14$ $\times 10^{6} \mathrm{~A} / \mathrm{cm}^{2}$, and the value of $\tau_{S}(j)$ for any selected value of $j$. If these selected $j$ are noticeably smaller than the value $0.83 j_{S}$ defined by Eq. (23) for the above-mentioned $u_{l}$ $=0.76$, we are dealing with the tunnel emitter current limitation and can use $\bar{E}=0.86 \times 10^{5} \mathrm{~V} / \mathrm{cm}$ as an approximate value of $E(0)=E(j)$. As a result, we can calculate $A_{0}(j)$ $\cong e \bar{E} \tau_{S}(j) / m V_{S} \quad$ [see Eq. (19a) $]$ and $\theta_{l}(j)=2\left\{\left[u_{l}^{1 / 2}\left(u_{l}\right.\right.\right.$ $\left.\left.+2)^{1 / 2}+A_{0}^{2}\right]^{1 / 2}-A_{0}\right\}$ [see Eq. (20)].

For calculations of $G\left(\chi_{l}\right)$ and $B\left(\chi_{l}\right)$, we also need to select the value of $\Omega_{0}$, which can be different for the same $A_{0}$. Note that the parameters $A_{0}=e \mathrm{E}(j)\left(\kappa_{D} / 2 e j m V_{S}\right)^{1 / 2}$ and $\Omega_{0}=d j /\left.d E\right|_{x=0}\left(m V_{S} / 2 e j \kappa_{D}\right)^{1 / 2}$ have the same origin and are determined mainly by the tunnel emitter properties. In this article, we do not investigate their interrelations and consider them as self-consistent independent parameters. In real samples, different values of $\Omega_{0}$ for the same $A_{0}$ or different values of $A_{0}$ for the same $\Omega_{0}$ can be provided both by the selection of materials for the $\mathrm{B}$ barriers and $\mathrm{C}$ contacts and by the selection of the B-barrier form and thickness. For example, we demonstrate in Fig. 7 (see the inset) three possible functions $j(E)$ crossing each other at the same point. At this point, they have the same values of $j, E$, and $A_{0}$. But the values of $\Omega_{0}$ for these three cases are substantially different.

We have calculated $G\left(\chi_{l}\right)$ and $B\left(\chi_{l}\right)$ for five values of $j$ $\left(=10^{4}, 5 \times 10^{4}, 10^{5}, 2 \times 10^{5}\right.$, and $10^{6} \mathrm{~A} / \mathrm{cm}^{2}$; as we saw above, the last value is really invalid and considered to show the trend and for comparison) and six values of $\Omega_{0}$ $(=\infty, 10,3,1,0.3$, and 0$)$. Five values of $A_{0}(=8.22,3.68$, $2.60,1.84$, and 0.82$)$ and five values of $\theta_{l}(=0.175$, $0.384,0.53,0.717$, and 1.279$)$ correspond to the abovementioned five values of $j$, respectively. All the results obtained are presented in Figs. 7-10. We see that the pictures presented in these figures are similar to each other and have some characteristic peculiarities.

(1) There exist regular windows of negative conductance for all the considered values of $j$ and $\Omega_{0}$. At $\Omega_{0}=\infty$, these windows are situated in the $\chi_{l}$ sections $((2 k-1 / 2) \pi,(2 k$ $+1 / 2) \pi), k=1,2,3, \ldots$. They shift to the low-frequency side with a decrease in $\Omega_{0}$. At $\Omega_{0}=0$, the negative conductance windows are approximately situated in the same sections where the positive conductance windows at $\Omega_{0}=\infty$ are placed.

(2) At $\Omega_{0}=0$, the lowest-frequency negative conductance window has the left boundary at $\chi_{l}=0$. This means that we can reach very low-frequency oscillatory regimes if we are able to implement the required small values of $\Omega_{0}$.

(3) The maximum negative conductance for small values of $\Omega_{0}$ is reached in the first window at $\chi_{l}=\chi_{l M} \cong \pi$. It decreases with an increase in $j$. The real frequency value is $f_{M}=\omega_{M} / 2 \pi=\Omega_{M} / 2 \pi \tau_{S}=\chi_{l M} / 2 \pi \tau_{S} \theta_{l} \cong 1 / 2 \tau_{S} \theta_{l} \approx 5 \mathrm{THz}$ since the product $\tau_{S}(j) \theta_{l}(j)$ weakly depends on the $j$ value. For large $\Omega_{0}$, both the frequency $f_{M}$ and the negative conductance $G_{M}=G\left(f_{M}\right)$ increase, and $f_{M} \cong 10 \mathrm{THz}$ at $\Omega_{0}$ $\rightarrow \infty$.

(4) The relation $|G| / B$ cannot be larger than $0.25-0.3$ in the first negative conductance window and becomes much smaller for the higher-frequency negative conductance windows $(k>1)$.

In Figs. 7-10, we have plotted both normalized values $\left(\chi_{l}=\Omega \theta_{l}, R_{S} G\right.$, and $\left.R_{S} B\right)$ and the same values in their "natural" units $\left[f(\mathrm{THz}), G\left(1 / \Omega \mathrm{cm}^{2}\right)\right.$, and $\left.B\left(1 / \Omega \mathrm{cm}^{2}\right)\right]$. 


\section{DISCUSSION}

In this article, we considered first the small-signal theory of a semiconductor heterostructural transit-time oscillator with ballistic (or quasiballistic) electron transport in the T space. We started with the simplest case when a current in the $\mathrm{T}$ space is limited only by the electron space charge and a conventional nonparabolic dispersion relation [see Eq. (13)] characterizes the electrons. Generally speaking, we expect that this nonparabolicity will lead to the elimination of the negative conductance windows. But we noticed that such elimination takes place for very high values of the voltage $u_{l}=e U_{l} / m V_{S}^{2}$. For moderate values of $u_{l}(\sim 3-6)$, which are the only ones possible in realistic material systems, the negative conductance windows can be successfully realized, and oscillators based on such regimes are possible. Note that, in comparison with vacuum diodes, not only much simpler designs and more effective electron emission mechanisms but also substantially smaller electron effective masses characterize semiconductor transit-time devices.

On the basis of the space-charge limited regimes considered, we turned to the case of the tunnel electron emission limited current. We assumed that the voltage drops mainly across the $T$ space and the emission current is controlled by the electric field value on the T-space side in the boundary B barrier/T space. We did not consider in detail the tunnel current characteristics $j(E(0))$ and assumed only that the characteristics are known and we can calculate the introduced parameters $A_{0}$ and $\Omega_{0}$, which depend on the direct current density $j$ and are determined by $E(j)$ and $d j /\left.d E\right|_{x=0}$, respectively. Of course, such a description does not allow us to calculate the detailed $U j$ characteristics. But it has allowed us to show that the correct selection of a tunnel emitter with an appropriate $E(j)$ dependence can substantially increase the negative conductance of the $\mathrm{T}$ space for moderate and high values of $u_{l}$.

All of the above-stated results were obtained assuming a nonreflecting and nonbackscattering $\mathrm{T}$ space/A contact boundary at $x=l$. This boundary should absorb the hot electrons coming from the $\mathrm{T}$ space entirely. Such a requirement is not unique for ballistic systems, which always need to have similar collector boundaries. The specificity is in the very high energy of the arriving electrons $(0.5-0.6 \mathrm{eV})$, which cannot dissipate in a short time. Therefore, these hot electrons, after scattering their momentum, have a realistic probability of coming back into the $\mathrm{T}$ space. We can imagine two simple methods to avoid this situation.

(1) The first method is to form a metallic contact with a well-extended Fermi surface directly behind the T space/A contact boundary in order that the hot electrons transfer their energy to the Fermi electrons. This situation is analogous to the situation in a metallic anode of a vacuum-tube device. It might be acceptable for single-transit-space oscillators, but is unsuitable for cascading oscillators.

(2) In cascading oscillators, which are necessary to obtain higher power and higher efficiency, each of the next $\mathrm{C} / \mathrm{B} / \mathrm{T} / \mathrm{A}$ cells should be grown over the previous $\mathrm{C} / \mathrm{B} / \mathrm{T} / \mathrm{A}$ cells. This means that the A contact must be a semiconductor and appropriate for the next growth of the $\mathrm{C}$ contact, etc. The simplest solution to this problem is to use a heavily doped multivalley semiconductor as the material for the A contact. Since the densities of states in the $L$ and $X$ valleys usually are much higher than the density of states in the $\Gamma$ valley, the energy scattering process across these valleys with the participation of both intravalley and intervalley phonons is much more intense than only across the $\Gamma$ valley. In addition, $L$ and $X$ electrons cannot go directly from $L$ and $X$ valleys in the A contact to the $T$ space where these valleys are emphatically absent in the energy range considered.

Unfortunately, InP and other materials that can be used in the $\mathrm{T}$ space have no ideal multivalley isomorphic counterparts to use for the A contacts. For such a role, an alloy isomorphic to $\mathrm{InP}$ such as $\mathrm{GaAs}_{0.5} \mathrm{Sb}_{0.5}$ (see Ref. 18) or, maybe, $\mathrm{GaP}_{0.35} \mathrm{Sb}_{0.65}$, is most suitable for the InP T space, and an alloy such as $\mathrm{GaAs}_{0.08} \mathrm{Sb}_{0.92}$ is most suitable for the $\mathrm{InP}_{0.69} \mathrm{Sb}_{0.31} \mathrm{~T}$ space.

One more method to use noncentral valleys is based on strain-balanced superlattices (SL's). Such SL's should include quantum wells, which contain noncentral valleys in the necessary energy range. For example, the InP/InAs heterojunction has a very large conduction band offset ${ }^{10}(0.75 \mathrm{eV})$. For such energies, $L$ valleys exist in InAs. This means that we can hope to reach the necessary effect by using a heavily doped strain-balanced GaAs/InAs SL as the A-contact material. One of the versions of the TUNNETT considered can be obtained for $\delta_{2}=0$, that is, $\delta_{1}=\delta_{3}$ (see Fig. 1) and the B barrier does not exist. In this case, the triangle barrier formed at the left edge of the $\mathrm{T}$ space serves as the tunnel barrier and the dependence $j=j_{0}\left(E_{0}\right)$ can be presented by the classic Fowler-Nordheim formula ${ }^{19}$

$$
j / j_{N}=\left(E / E_{N}\right)^{2} \exp \left(-E_{N} / E\right),
$$

where $J_{N}$ and $E_{N}$ are parameters that depend on the electron effective masses in the $\mathrm{C}$ contact and $\mathrm{T}$ space and also on $\varepsilon_{F}$ and $\delta_{1}$. The dependence $E(j)$ in Eq. (19a) can be obtained by inversion of this formula.

\section{CONCLUDING REMARKS}

We have shown in this article that transit-time diodes with ballistic electron transport in the $\mathrm{T}$ space are interesting as possible oscillators for the terahertz range. For a successful implementation of such oscillators, several additional problems need to be addressed.

(1) To obtain explicit expressions for the parameters $A_{0}(j)$ and $\Omega_{0}(j)$ introduced in this article, which depend on the dc current density $j$ directly and the field $E(x=0)$ $=E(j)$, we need a correct theory of tunnel emitters with a detailed description of both $\mathrm{C}$ contact and $\mathrm{B}$ barrier.

(2) To provide nonreflecting and nonbackscattering A contacts, we need to develop a more detailed A-contact design and a more detailed theoretical description of these contacts.

(3) To solve the cascading oscillator problem, we need a more detailed design of the intermediate A-C contacts.

In the next stages, we need to develop a nonlinear description of the oscillatory regimes and a theory of oscillator diodes in realistic resonators. 


\section{ACKNOWLEDGMENTS}

The writers thank Dr. S. Tipton for numerous useful notes. This work was supported by the AFOSR through the MURI program F 49620-00-1-0328 and NSF grants including ECS-0099913.

${ }^{1}$ J. Nishizawa and Y. Watanabe, Sci. Rep. Res. Inst. Tohoku Univ. A 10, 91 (1958).

${ }^{2}$ V. K. Aladinski, Sov. Phys. Semicond. 2, 517 (1968).

${ }^{3}$ J. Nishizawa, K. Motoya, and Y. Okuno, IEEE Trans. Microwave Theory Tech. 26, 1029 (1978).

${ }^{4}$ H. Eisele and G. I. Haddad, IEEE Trans. Microwave Theory Tech. 46, 739 (1998).

${ }^{5}$ G. I. Haddad, J. R. East, and H. Eisele (unpublished).

${ }^{6}$ C. Kidner, H. Eisele, and G. I. Haddad, Electron. Lett. 28, 511 (1992).

${ }^{7}$ H. Eisele and G. I. Haddad, IEEE Trans. Microwave Theory Tech. 42, 2498 (1994).
${ }^{8}$ H. Eisele and G. I. Haddad, IEEE Trans. Microwave Theory Tech. 43, 210 (1995).

${ }^{9}$ T. Bauer, M. Rosh, M. Claassen, and W. Harth, Electron. Lett. 30, 1319 (1994)

${ }^{10}$ I. Vurgaftman, J. R. Meyer, and L. R. Ram-Mohan, J. Appl. Phys. 89, 5815 (2001)

${ }^{11}$ S. Adachi, J. Appl. Phys. 61, 4869 (1987).

${ }^{12}$ M. V. Fischetti, IEEE Trans. Electron Devices 38, 634 (1991).

${ }^{13}$ S. Tiwari and D. J. Frank, Appl. Phys. Lett. 60, 630 (1992).

${ }^{14}$ J. Bude and K. Hess, J. Appl. Phys. 72, 3554 (1992).

${ }^{15}$ W. E. Benham, Philos. Mag. 11, 457 (1931).

${ }^{16}$ F. B. Llewellyn and A. E. Bowen, Bell Syst. Tech. J. 18, 280 (1939).

${ }^{17}$ Handbook of Mathematical Functions, edited by M. Abramowitz and I. A. Stegun (Dover, New York, 1965)

${ }^{18}$ J. Hu, X. C. Xu, J. A. H. Stotz, S. P. Watkins, A. E. Curzon, M. L. W. Thewalt, N. Matine, and C. R. Bolognesi, Appl. Phys. Lett. 73, 2799 (1998).

${ }^{19}$ R. H. Fowler and L. Nordheim, Proc. R. Soc. London, Ser. A 119, 173 (1928). 